\title{
Research on Power Supply Method Optimization of PLC Control System
}

\author{
Wulin He \\ Liaoning Railway Vocational and Technical College, Jinzhou, 121013, China \\ 416198816@qq.com
}

Key words: PLC control system; power supply; optimize

\begin{abstract}
The rapid development of science and technology has provided more efficient ways for the intelligent industrial control management. As one of the popular control systems, PLC control system plays an important role in determining the control of whole power supply system. The stability of power supply system determines the actual effect of control system. The design of the whole power system should be started from the actual direction, especially for PLC that needs to contact the specific power requirements to provide more reasonable control for control system and the independent electricity system of PLC and power supply redundancy of external devices when the control system does not allow power disconnected, etc. Based on the detailed analysis of PLC, this paper optimizes the working process of control system to provide a more stable power supply system.
\end{abstract}

\section{Introduction}

The development of industry provides a broader market and higher requirements for automation equipment. But continuous production puts forward higher requirements on power safety and quality of automation equipment.

In practical project, scientific and reasonable power supply system is an important guarantee for the smooth operation of the project and the material base for the project completion. PLC control system as a management tool in the new era, its operation does not need too stringent measures, but the the design requirements of power supply system is more and more high as the advantages of technology upgrading. In the process, PLC control system has a far-reaching influence on the whole control system and power supply system. The traditional control method can not meet the needs of production, and the corresponding automatic control technology has been widely used. However, the large integrated control system is increasingly demanding for power systems. In order to ensure the reliability of the automatic control system, the reliability and stability of the power supply system must be guaranteed first. In this demand, uninterruptible power supply (ups) has been widely used. In the process of using, traditional UPS had been found that it has many defects in the implementation process, so the targeted optimization was carried out.

\section{Power supply design and grounding of PLC control system}

Power supply design:

Since the PLC power supply circuit is for ac22v 0 and 50zh general city Electric-capacity, the power grid frequency should be as stable as possible during normal operation.

In the power supply network, the frequent start-stop equipment phenomenon frequently happens to big consumers, for this reason, the power grid accidents should be avoided to the greatest extent. According to the operating state of PLC, specific defensive measures are necessary in order to 
improve the effectiveness of the control system and prevent interference of external factors on the circuit. No matter electromagnetic interference or signal interference, it should be started from the perspective of power supply protection. But in PLC system, it is connected to the overall system both power supply protection and power supply instrument lights, so when system unstable situation is happened during normal operation, which can cause electromagnetic interference gets into PLC control system through the impedance and the path of power line, then unstable factors are caused within the system, so in this case, internal disturbances can cause instability. In the design of PLC control system, it should be started from the specific design requirements, considering the unexpected factors that are the important goals causing unstable system. For PLC system, in order to realize the system internal device and the normal operation of each module, it needs isolating process on the protection of the power supply, the wide spectrum of power grid disturbance can produce interference to PLC control system and other units.

Ground handling:

In PLC control system, it is reasonable to distinguish grounding modes among digital, communication, shielding, signal, analog, etc for the sake of service security. The connection of the ground line in the project shall be based on the following principles: replace the grounding with a special grounding or common grounding; separate the signal from the ground wire; separate the connection between the shielding copper row and the grounding copper row; connect PLC operation manual. When PLC leaves the factory, it fully takes into account all kinds of anti-interference abilities, whose reliability is at a level. However, in the process of industrial control, it makes electromagnetic interference very serious because of complex start-stop process and the entanglement of power line and control line, especially the complexity of the input and output channels in the industrial control system makes the distance between the line and the line widening. The unfortunate combination also forms channel interference. In addition, if PLC control system has a joint position, the differences in position are due to the potential difference of the ground ring current position, which results in serious common mode interference. The grounding anti-jamming technology is the solution to a series of technical problems centered on the ground ring current. It can effectively reduce and suppress the interference of the ground loop current through a small amount of common ground. The electric field interference and magnetic field interference caused by input and output channels are different, so are the corresponding solutions. The distribution interference between two conductors of power plants is mainly caused by two modes: remote interference and shielding interference. Distance means the weak signal line is laid away from the signal line, especially the power line, meanwhile the safety distance between the wires is ensured more than $100 \mathrm{~mm}$. Avoid parallel lines or shorten the length of parallel lines as much as possible. Shielding refers to shielding the target conductor and electric field in a hollow conductor or metal net. In the field of industrial control, reactors, large motors, magnetic switches and large current are all strong magnetic interference sources. It is the most effective method stopping interference phenomenon from the source, but considering the actual production process and implementation difficulties, which needs another technology to deal with problems on the basis of the original foundation, so as to improve the stability of the whole system. Keeping away from technology is an effective way to eliminate distractions. Avoiding parallel lines or shortening the length of parallel lines can also reduce magnetic interference. The grounding interference technology can really help PLC control system to operate normally. In the process of the original design, it is needed to consider a variety of accidents to reduce the degree of external interference to the design of PLC control system, which can protect the power supply system on the whole. This is also a part of the current design process that requires active participation. 
The grounding design is an important part of the design, which requires the protection and design of the circuit. Firstly, arranging ground wire system reasonably. Ground wire of PLC system can be divided into logical ground wire of digital circuit, analog ground wire of analog circuit, relays, noise ground wire of motor and another high-power electrical equipment and shielding grounding wire of instrument housing, etc. These grounding wires shall be arranged separately but connected to a power source at a point. Secondly, choosing a variety of grounding methods. The more practical circuit requirements and signal frequency should be adjusted in the specific application environment, which are helpful to make the influence of circuit system reduced in the process of signal frequency changes, and adopt different ways of grounding according to the different grounding impedance. The differences between single point grounding and multipoint grounding decide impedance. Thirdly, the length and thickness of the ground wire are considered. The larger the thickness is, the less the influence of the impedance will be.

\section{Power distribution of PLC control system}

Distribution of power in groups:

In the actual production work, the power line will cause great interference to the work situation. When designing and optimizing the power line, the influence of such interference should be reduced as far as possible. It is the most effective way to realize the two phase power supply by dividing the equipment with greater interference into a measuring control device, using different power lines and using the shielded cable in the distribution room.

The independent power supply of the measurement and control device and the power equipment:

By adjusting the power supply status of the device and module in the measurement and control device, the degree of external interference can be reduced, but this also affects the standard of load exchange, increases the resistance to the external interference, and easy to appear unstable neutral point under the asymmetrical load.

The power demand of the job requires is small, so it can choose a power which has less interference and does not affect the normal operation of the system. In actual operation, power distribution boxes or power transformers can be used to supply power according to specific conditions.

Select reasonable of power capacity of power supply:

The large reserves of the whole power supply can make the measurement and control device adapt to the wide range of load changes, prevent the internal interference caused by the power supply, and increase its dynamic performance.

Therefore, the increase of power supply is closely related to capacity and cost, so we usually choose 0.5 - 1 times.

\section{Power isolation of PLC control system}

Noise will interfere with the actual control process, so strengthen the stability of the control system and adjust the AC power in disturbed condition to adapt to noise interference. Separating the measurement and control system from the power supply can avoid the common resistance caused by the disaster, strengthen the stability of the power supply system and reduce the sensitivity to the fluctuation. In this case, the 1: 1 isolation transformer is added to the power transformer and the low power amplifier.

At present, NCT developed abroad can control noise voltage and common mode noise voltage which is an ideal isolating transformer. In the optimized power supply scheme, the DC-DC system 
can also be used to isolate DC power to enhance the system's ability to suppress electromagnetic interference. If the control system needs to be separated from the internal subsystem of the electrical equipment, the DC power supply should be provided separately.

In order to meet the actual requirements of all parts of the power supply system, and to strengthen the effectiveness of the power supply, it is necessary to protect and improve all parts of the equipment so as to improve the safety of the whole work equipment in order to make a reasonable configuration of the power supply to ensure the security of the whole system. The field instrument generally has no influence on the transient power of the power supply, but it has an influence on the transmission signal involved in interlocking, so it is necessary to improve the quality of power supply in the actual work.

\section{Power redundancy technology of PLC control system}

The AC power supply adopts double redundant power supply, which can improve the stability of the power supply system. The two way is to separate different substations, and automatically switch to another line when there are problems. Uninterruptible power supply (UPS) can effectively guarantee the reliability of computers. However, the aging of UPS's own electrical equipment will result in failure. In order to ensure its stable operation, the ups redundancy technology can be used to connect the ups double machine hot standby and the standby machine to the host. When the AC power is cleaned, it can access two UPS AC power input terminals. The dual machine hot standby ensures the load equipment to be jammed when AC power interrupts so as to avoid production data, equipment, systems and other problems. In order to improve the stability and reliability of the DC power supply system, two DC power sources can be cleaned with diode ice to ensure the normal operation of the system when a DC power supply fails. We should take into account the design requirements of the field environment when selecting the diode, and diode types tend to be consistent so as to prevent the conflict from causing the hidden danger of the line, in this way, we can make the two power supply consistent and ensure the effective processing of the diode fault. First, air switch of the controller circuit is optimized, and the original single switch is changed to double parallel-control switches to prevent the controller from being cut off due to the damage of the air switch. Then, the $24 \mathrm{~V}$ DC power supply circuit is optimized, the first two $24 \mathrm{~V}$ DC power supply parallel operation can have a standby power supply. When the power supply box fails, the power box is automatically switched to another power supply, and the damaged power box will automatically cut off the system. Through the field equipment test of the EJA pressure transmitter, the field equipment will not stop running during the switching of the power supply, so that the production process will not be affected to reduce production.

The design of the control system itself revolves around the power supply, so any design should start with safe power supply, reduce the unexpected effects of all aspects on the power supply system while providing stable power supply, and prevent the hidden danger of unstable use of the power supply system caused by the conflict between the power supply system and the equipment.

\section{Conclusion}

The power optimization function of PLC control system is essential when taking into account the stability and reliability in the process of system design. In the process of system design, stability and reliability should be taken into account. The power optimization function of PLC control system is essential. According to the environment and characteristics of the practical application 
system, flexible selection can effectively improve the efficiency of power supply technology. Because of the popularization and application of industrial automation control application platform, the pace of self-reliance has been accelerated. Industrial enterprises are more and more dependent on the network computer system. With the continuous progress and development of the times, the demand for PLC control technology will become more and more large, which will further promote the stability and efficiency of industrial production activities, and boost the continuous progress of the process of industrial production automation in China.

\section{References}

[1] Zhang Lijian. Interference Analysis and Optimization Control of PLC Control System for Bucket Wheel Stacker Reclaimer [J]. Electronic components and information technology, 2017, 1(02): 76-78.

[2] He Ye. Application of PLC in Plant Power Supply Automation System [J]. Computer products and circulation, 2017 (08): 251.

[3] Zhao Xiang. Research on Design, Construction and Application of PLC in Metro 0.4KV Power Supply System [J]. Science and Enterprises, 2016(08): 103-104.

[4] Yang Weibin. Research on the Reliability of PLC Control System[J]. Digital Technology and Application, 2015(11): 7.

[5] B. Kai, W. J. Chen, L. T. Wang, H. B. Shen, R. C. Li, Y. L. Wang, H. J. Zhao, "High-speed railway traction power supply contact network lightning protection.," Journal of Chinese electrical engineering, vol. 10, pp. 191-199, 2013.

[6] L. J. Zhou, F. Gao, R. F. Li, X. B. Cao, G. N. Wu, "Lightning protection system of high speed railway traction power supply system.," High voltage technology, vol. 02, pp. 399-406, 2013. 